\title{
Pratiques
}

Linguistique, littérature, didactique

$169-170$ | 2016

Enseignement/apprentissage de la langue, des textes et des discours. 40\&nbspans de Pratiques

\section{Pour un apprentissage de la réflexion métalinguistique à l'école élémentaire}

For a Learning of Metalinguistic Reflection in Elementary School

Lionel Audion

\section{CpenEdition}

Journals

Édition électronique

URL : http://journals.openedition.org/pratiques/3050

DOI : 10.4000/pratiques.3050

ISSN : 2425-2042

Éditeur

Centre de recherche sur les médiations (CREM)

Référence électronique

Lionel Audion, "Pour un apprentissage de la réflexion métalinguistique à l'école élémentaire »,

Pratiques [En ligne], 169-170 | 2016, mis en ligne le 30 juin 2016, consulté le 19 avril 2019. URL : http:// journals.openedition.org/pratiques/3050 ; DOI : 10.4000/pratiques.3050

Ce document a été généré automatiquement le 19 avril 2019

(C) Tous droits réservés 


\title{
Pour un apprentissage de la réflexion métalinguistique à l'école élémentaire
}

\author{
For a Learning of Metalinguistic Reflection in Elementary School
}

\author{
Lionel Audion
}

1 Dès le colloque de Cerisy organisé par Pratiques en 1979, J.-F. Halté et A. Petitjean appelaient à un «nouvel enseignement du français». Appel souvent relayé depuis, notamment par B. Combettes, J.-P. Lagarde, J.-P. Bronckart, C. Vargas...

2 Or, nous constatons que, dans le premier degré, la reconfiguration attendue n'a pas eu lieu en grammaire, c'est ce que nous rappellerons dans une première partie: la terminologie s'est sans doute modernisée, mais les pratiques de classe, transmissives, et les référents linguistiques ont peu évolué ces trente dernières années; la grammaire de phrase et le classificatoire y règnent en maitres, l'énonciation est ignorée, de même que les zones de flou, décrites par B. Combettes. Quant aux corpus proposés, la doxa est celle $\mathrm{du}$ "texte authentique »: la langue scolaire est écrite et datée, elle ne reflète pas la richesse de la langue ordinaire des élèves, c'est un « champ opératoire aseptisé » selon A. Culioli (Bouquet, 2004, p. 138). L'exercice phare est celui de la transformation de phrases mais sans réelle réflexion sur cette opération. Enfin, les connaissances «déjà-là » des élèves sont souvent ignorées.

Nous proposerons donc, dans une seconde partie, une approche à contrecourant prenant en compte la sémantique en plus de la syntaxe et de la morphologie, s'appuyant sur une théorie des observables, proposant donc des corpus construits et non "authentiques", considérant les opérations d'énonciation et pas seulement le cadre phrastique de la langue écrite scolaire, et proposant des opérations de transformation d'énoncés, mais dans le but de faire apparaitre un problème linguistique sur lequel les élèves réfléchiront à partir de leurs connaissances épilinguistiques. Nous inscrivons donc nos travaux dans le cadre de la Théorie des opérations énonciatives (TOÉ) conçue par A. Culioli, dont nous 
essayons d'adapter la démarche à l'apprentissage de la langue 1 en école élémentaire, sans en proposer pour autant une "transposition didactique». Il ne s'agit pas en effet pour nous d'adapter l'ensemble de la TOÉ pour en en faire une nouvelle grammaire scolaire, ce ne serait ni souhaitable ni possible, mais d'en retenir quelques points saillants, dans l'approche linguistique (faire «travailler les énoncés» pour faire apparaitre un problème de langue) et dans les notions (par exemple le domaine notionnel, mais aussi les notions d'ajustement, de coénonciateur...).

Pour ce faire, nous avons forgé un outil, testé en classe, permettant une problématisation rapide d'un fait de langue : le carré d'énoncés, et un rituel : le débat d'acceptabilité.

\section{De la reconfiguration souhaitée dans les années 80 à l'urgence de la reconceptualisation}

5 Le constat a souvent été fait que la grammaire scolaire ne répond pas aux besoins des élèves et que de nombreux enseignants du primaire l'enseignent sans lui donner de sens, sans se poser de questions sur son utilité et sans connaissances théoriques précises. Nous savons depuis longtemps que l'essentiel de cet enseignement est une invention scolaire qui ne repose pas sur un fondement théorique clairement défini (Chervel, 1977), mais l'habitude s'est installée, comme une exception française, et l'on continue pourtant en parallèle à se lamenter sur le fameux « niveau » des élèves, qui ne cesserait de baisser.

\subsection{La volonté de reconfigurer l'enseignement grammatical}

6 Un colloque a été organisé dès 1979 à Cerisy par les didacticiens J.-F. Halté et A. Petitjean ; il avait pour but de faire le point sur l'entrée de la linguistique à l'école suite au plan «Rouchette » de rénovation de l'enseignement du français, et concluait à la nécessité d'une nouvelle didactique. Les spécialistes de la langue savaient déjà que la linguistique avait été intégrée trop tôt et de manière précipitée aux programmes scolaires: les travaux de N. Chomsky, par exemple, n'étaient pas encore suffisamment maitrisés par les formateurs d'enseignants ni même, peut-être, par les concepteurs des programmes. Les enseignants les plus anciens se souviennent encore des fameux "arbres de Chomsky" utilisés dès le CE2 pour expliquer aux élèves la construction de la phrase. Mais sans réelle réflexion sur l'ensemble de la théorie chomskyenne, cette approche, qui aurait pu être mieux didactisée, n'a pas remplacé la précédente : elle s'y est agglutinée pour accoucher d'un nouveau monstre grammatical.

7 En 1989, J.-P. Bronckart faisait à nouveau des propositions pour une nouvelle didactique du français, s'appuyant sur la langue telle qu'elle est réellement parlée et écrite dans les différentes situations d'énonciation que peut ou pourra vivre l'élève ; il s'agissait pour lui, citant les propos de G. Schoeni, «d'abandonner l'attitude normative qui ignore la langue de l'enfant au nom de la croyance en un français homogène et unique par rapport auquel tout serait déviance » (Bronckart, 1989, p. 61).

8 Malgré cet appel fort et largement relayé auprès des formateurs et des enseignants, les pratiques n'ont malheureusement guère changé, au point que S. Meleuc et N. Fauchart (1999) n'hésitaient pas dix ans plus tard à rappeler que la grammaire scolaire, particulièrement la conjugaison, représentait l'un des «noyaux durs de l'immobilisme » dans l'ensemble du paysage didactique. 
Pendant toute cette période, le didacticien C. Vargas n'a pas cessé d'appeler, lui aussi, à une transformation de l'enseignement de la grammaire; en 2009 encore, il dénonçait «Les incertitudes, les contradictions, les incohérences, voire les aberrations issues de la recomposition didactique des années 70-80 [et appelait à] une reconfiguration didactique des savoirs grammaticaux, qui permettrait de faire de la grammaire scolaire un objet cohérent ». (Vargas, 2009, p. 32).

\subsection{La nécessité de reconceptualiser l'enseignement grammatical}

L'appel de C. Vargas a été entendu, mais la reconfiguration est restée en surface: quelques ajustements terminologiques ont eu lieu, le regard sur la langue a évolué chez certains enseignants et donc, peut-être, chez leurs élèves, la pédagogie s'est déportée un peu du déductif vers l'inductif, mais il aurait fallu accompagner cette reconfiguration d'un ambitieux programme de formation de formateurs et de formation continue des enseignants, notamment dans le premier degré ou les maitres, polyvalents, ont plus de difficultés à tenir à jour leurs savoirs didactiques. De plus, ces changements ne se sont pas suffisamment appuyés sur des concepts issus des théories linguistiques et présentés comme tels; nous sommes donc d'accord avec J.-P. Bronckart (2014, p. 37) lorsqu'il écrit :

Dans la quasi-totalité des documents didactiques en usage, cette reconfiguration s'effectue [...] en neutralisant (ou en ignorant) les options épistémologiques soustendant les paradigmes théoriques, ce qui constitue à nos yeux une source de graves difficultés, en particulier dans le domaine de la formation des enseignants. On a donc continué à enseigner la langue française, en langue 1, en s'adossant sur une grammaire scolaire qui ne dit pas d'où elle vient ni quels paradigmes théoriques elle a retenus, mais en proposant un amalgame de plus en plus indigeste.

C'est pourquoi notre proposition didactique s'inscrit dans un cadre théorique fort et dûment référencé, afin de fonder l'apprentissage de la grammaire sur un « effort de réanalyse et de reconceptualisation », selon les vœux de J.-P. Bronckart (cf. « Que faire de la grammaire et comment en faire?", dans ce numéro).

\subsection{Principales lacunes du modèle actuel}

13 Si ce changement didactique est réclamé si fortement (et pourtant en vain), c'est que le modèle toujours en vigueur présente de nombreuses lacunes, dont nous rappellerons les principales pour mémoire.

14 a) La grammaire scolaire est presque exclusivement classificatoire ; on en connait les conséquences, mais on n'insiste peut-être pas suffisamment sur le fait que l'une d'entre elles est la mauvaise préparation des élèves à l'apprentissage d'une langue étrangère. Or, les enfants commencent cet apprentissage dorénavant très tôt, parallèlement à l'enseignement de la grammaire du français : si l'un et l'autre étaient complémentaires, au lieu d'être ressentis comme complètement différents, ce serait une grande avancée. Par exemple, le travail en grammaire française sur la détermination (cf. plus loin) ne dit rien de l'opération de détermination en elle-même ; les élèves apprennent que le pluriel de un/une est des (en ignorant que, souvent, ce pluriel est les) et, lorsqu'ils doivent s'exprimer en anglais, ne savent pas comment traduire des, car ils n'ont pas réfléchi à son sens ni à son rôle par rapport au nom ainsi déterminé. 
15 b) La grammaire scolaire est circonscrite à la phrase écrite, et cela dans le cadre d'une langue presque spécifique, «découpée » dans la langue française comme un patron en couture : une langue que, finalement, on ne rencontrerait que rarement à l'extérieur de l'école, ce qu'A. Culioli appelle « un champ opératoire aseptisé » (Bouquet, 2004, p. 138).

c) Les savoirs grammaticaux que les élèves se sont construit seuls, progressivement, ceux que F. Calame-Gippet (2008) appelle les "connaissances ignorées", ne sont presque jamais pris en compte par l'école. Pourtant, lorsqu'un enfant de maternelle raconte qu' " un loup *coura après les petits cochons ", un énorme travail de grammaire du texte et de morphologie du verbe a déjà été réalisé (l'enfant a par exemple compris comment se construit le passé simple par rapport à un modèle fréquent et à quel moment du récit il faut l'utiliser).

d) La grammaire scolaire fait semblant, depuis les années 80, de reposer sur une démarche inductive et même parfois sur une démarche de problématisation. Mais les séances d'étude de la langue les plus souvent pratiquées, celles, fréquentes, auxquelles nous assistons nous-même régulièrement en tant que formateur, ne pointent aucun problème de langue, font observer aux élèves des phrases «authentiques " prélevées presque au hasard ici ou là, au lieu de construire un énoncé que les élèves pourraient faire proliférer en le transformant au moyen d'une consigne simple, afin de bâtir un problème. Ceci interroge aussi bien entendu la pratique des «manipulations » d'énoncés proposées aux élèves, sans que, bien souvent, l'objectif problématique de ces manipulations ait été réellement réfléchi, à l'inverse des "proliférations" (gloses, paraphrases) que propose la TOÉ par exemple.

Un autre modèle théorique est donc nécessaire pour éviter tous ces écueils.

\subsection{L'évidence de la grammaire énonciative}

19 Le cadre théorique que nous avons choisi est celui de la grammaire énonciative, afin de permettre aux élèves d'observer la langue réelle telle qu'elle se parle et s'écrit dans des échanges, ce qui prend en compte le coénonciateur (on parle ou écrit avec ou pour quelqu'un qui n'est pas qu'un "récepteur» mais qui coconstruit l'énoncé) et les ajustements que cette prise en compte nécessite (je dis ce que je dis en fonction de ce que je sais ou crois savoir de la personne à qui je m'adresse; je procède donc à des ajustements en fonction d'elle et elle-même ajustera mon message en fonction de ce qu'elle sait ou croit savoir de moi). Notre but ici n'est pas de décrire cette grammaire ; on se reportera aux trois volumes d'articles d'A. Culioli (1990-2000) pour une description complète de la TOÉ, en particulier pour le rôle du coénonciateur. Nous avons choisi de développer ici l'exemple de la détermination, puisque ce sera également l'exemple retenu pour illustrer notre outil didactique, les Ateliers d'Antoine, en fin de deuxième partie.

\subsection{L'exemple de la détermination : comment la reconceptualiser}

En grammaire scolaire traditionnelle, cette notion est vue en CE1 et en CE2 avec un double objectif :

- Classer, étiqueter : d'un côté les articles définis (le, la ; pluriel : les) et de l'autre les indéfinis ( un, une ; pluriel : des).

- Insister sur l'accord déterminant-nom, à des fins orthographiques. Cet « enseignement » est assez vain, car il s'adresse à des enfants de huit ans qui ont automatisé depuis longtemps cet 
accord. Si certains d'entre eux font encore quelques erreurs, par exemple « *une cheval», alors qu'ils ont pris l'habitude depuis des années de faire l'accord en genre et en nombre, c'est parce qu'ils croient que « cheval » est féminin comme «balle », par exemple.

21 Le sens donné à ces articles est toujours le même : l'article indéfini sert à déterminer le nom la première fois qu'on le rencontre, et le défini sert dès la seconde fois : «J'ai vu un chien dans mon jardin et j'ai appelé mon père pour le faire sortir. Mon père a parlé fort et le chien est parti ${ }^{1}$. Les élèves "apprennent " également que le défini sert aussi à déterminer ce qui est censé être déjà connu de façon générale et pas seulement dans la phrase ou le texte : «Le Président de la république ; le directeur de l'école, etc. ».

Ces leçons de grammaire n'apportent rien aux élèves qu'ils ne savent déjà, au sens épilinguistique de ce terme ; elles sont suivies d'exercices qu'ils réussissent facilement et la notion, inscrite au programme, est ainsi traitée sans grande difficulté, ni pour le maitre, ni pour les élèves. Or, on n'a cependant rien dit de ce que signifie " déterminer » un nom. Quel est ce concept? Cette opération est pourtant très importante dans le langage, et elle ne se manifeste pas de la même façon dans les différentes langues. Ainsi, l'extraction au pluriel se traduit par l'emploi de des en français (j'ai vu des garçons et des filles) et par l'article zéro en anglais (I saw boys and girls). Ce qui compte, c'est donc que l'enfant comprenne cette opération, et ensuite voie comment elle se manifeste dans telle ou telle langue dont, bien sûr et prioritairement, le français.

Dans les théories énonciativistes, on préfère s'intéresser aux opérations, dont les mots sont les traces, c'est pourquoi il est souhaitable d'expliquer aux enfants les opérations d'extraction, de fléchage et de parcours. Certes, il ne sera pas question d'employer ces termes-là avec des enfants de 8 ou 9 ans, mais il est possible de les leur expliquer simplement :

24 a) L'extraction consiste à extraire une occurrence d'une notion. Expliquons aux enfants que le nom «fleur» n'existe pas dans la langue... Surprise des élèves qui sont bien convaincus du contraire! Pourtant, en langue, on trouve " une fleur », " la fleur », " ma fleur », etc., mais presque jamais «fleur» (à l'exception des apostrophes, des titres, des légendes d'images, etc.). Cette notion-là ne se trouve que dans le dictionnaire ; pour lui «donner vie » il faut extraire une occurrence de la notion «prélinguistique » fleur, au moyen de un ou une. Les mots prennent vie en s'envolant du dictionnaire.

b) Lorsque je veux utiliser un nom qui est "déjà là ", car il a déjà été créé dans la coénonciation (opération de "fléchage»), j'utilise l'article défini ou un autre déterminant. Contrairement à ce que dit souvent le discours scolaire, l'occurrence « déjà là » n'a pas forcément été rencontrée « avant » dans le texte ou dans un contexte général (comme «le président de la république»), mais elle peut être connue des seuls coénonciateurs: je peux par exemple dire «ce soir, je vais chercher les enfants à l'école » : mon coénonciateur sait de quelle école il s'agit, celle qui existe "déjà » dans l'espace de la coénonciation.

c) Enfin, je peux parcourir l'ensemble des occurrences de la notion, sans m'arrêter sur une en particulier; en français, on utilise dans ce cas-là l'article défini (« la rose est une belle fleur») ou indéfini (" une rose sent toujours bon»). Les deux articles sont-ils interchangeables ? Correspondent-ils à la même opération? Voilà un problème pour le linguiste ; je m'aperçois que je peux rencontrer deux un différents : celui de l'extraction et celui du parcours ; bien que la grammaire scolaire affirme que le pluriel de un est des, ces deux un ont-ils le même pluriel ? Voilà un exemple de problème posé au didacticien. 


\section{La réponse du didacticien : les Ateliers d'Antoine, une approche à contrecourant}

27 Les Ateliers d'Antoine, proposés pour le cycle $3^{2}$, sont inspirés des méthodes d'A. Culioli pour faire " proliférer les énoncés » et de sa réflexion sur les opérations énonciatives. Sa théorie est considérée comme "ouverte»: des chercheurs successifs ont contribué à l'enrichir, et le volant didactique (en langue seconde) a déjà donné de bons résultats : «Chaque fois que [mes théories] ont été, non pas utilisées, mais adaptées, aménagées, en particulier dans le domaine de la linguistique appliquée à l'enseignement des langues, ça s'est révélé extrêmement fructueux » (Culioli, 2005, p. 233).

28 Notre démarche consiste à proposer aux enseignants des fiches (une douzaine sont réalisées pour le moment et ont été testées en classe) contenant l'atelier à destination des élèves et les « savoirs du maitre ", résumant l'essentiel de ce que les enseignants doivent connaitre pour aborder l'atelier.

\subsection{Des « savoirs pour l'action » destinés aux enseignants débutants}

Plutôt que d'expliquer cette didactisation de la théorie des opérations énonciatives en formation initiale, nous nous sommes inspiré de la démarche proposée par R. Goigoux et S. Cèbe $(2007 ; 2011)$ qui proposent aux chercheurs de construire des outils didactiques à partir de leurs résultats de recherche, d'accompagner leur mise en œuvre dans des classes-tests, d'améliorer l'outil en fonction de la façon dont les enseignants se l'approprient ou non: "Nous pensons [...] plus efficace d'agir directement sur les pratiques des enseignants pour modifier leurs conceptions, plutôt que l'inverse. »

Il s'est donc agi pour nous, formateur en ESPÉ, d'étudier auprès des professeurs-stagiaires et néotitulaires ce que P. Rabardel (1995) appelle «la genèse instrumentale »: les enseignants vont-ils transformer l'artéfact que nous leur proposons en instrument? Vont-ils se l'approprier? Des observations en classe, des séries d'entretiens, permettent d'évaluer cette appropriation.

31 Afin de réunir l'exigence de J.-P. Bronckart (cf. plus haut) d'ancrer la reconceptualisation dans une théorie claire, et celle de R. Goigoux, nous avons décidé de joindre à chaque atelier «élève» une fiche "savoirs du maitre» qui récapitule aussi clairement et succinctement que possible les savoirs théoriques indispensables aux enseignants; ces informations décrivent le phénomène de langue problématisé dans l'atelier proposé aux élèves, en faisant clairement référence à la théorie culiolienne dont les grandes lignes ont été résumées dans la première fiche "savoirs du maitre». Par exemple, ce qui a été rappelé ci-dessus concernant la détermination (les opérations d'extraction, fléchage et parcours) est explicité dans un langage que des enseignants polyvalents, qui n'ont pas de bagage linguistique ou très peu, peuvent comprendre.

La fiche destinée aux élèves, quant à elle, est constituée d'une phase de problématisation rapide d'un fait de langue, le "carré-problème», et d'une proposition de débat métalinguistique entre élèves à propos des solutions apportées au problème. 


\subsection{Le dispositif didactique proposé : un outil et un rituel}

\section{en présence d'un problème de langue. À titre d'exemple, voici le carré-problème proposé} pour l'un des Ateliers dédiés à la question de la détermination:

Figure . La détermination

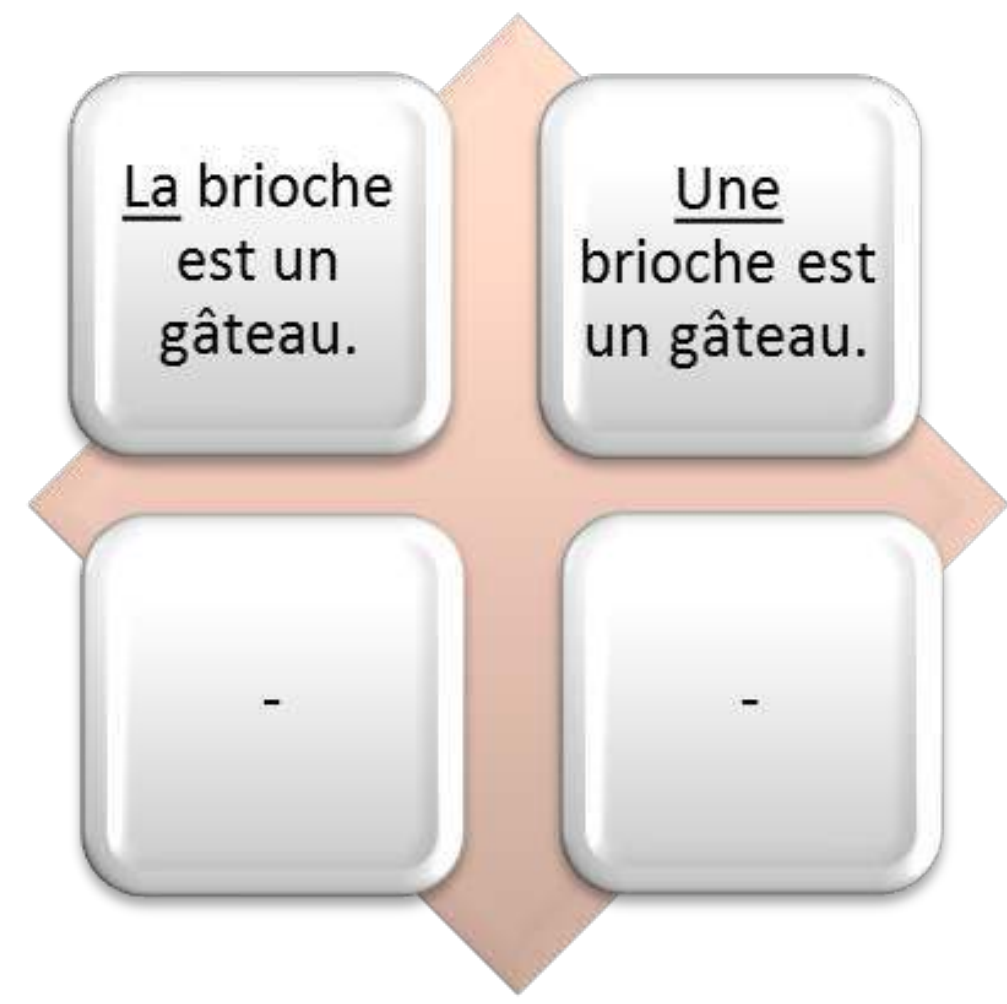

Transformer le premier énoncé ne pose pas de difficulté : tous les élèves proposent « les brioches sont des gâteaux » et peuvent ainsi appliquer la leçon qu'ils ont apprise : le pluriel de la est les et celui de un est des. Le second, en revanche, met les élèves face à un conflit de loyauté : les uns, les élèves les plus scolaires, proposent « Des brioches sont des gâteaux »: une devient des, et un devient des également. Mais à chaque fois que nous avons testé ou fait tester cet atelier, un ou plusieurs élèves proposent également «Les brioches sont des gâteaux », comme pour le premier énoncé. Le maitre, animateur de l'atelier, lance alors la deuxième étape : le débat métalinguistique : «nous sommes face à un problème, qui a raison et pourquoi ?» Chacun y va de ses arguments : les uns récitent leur leçon « le pluriel de une est des », les autres rétorquent : « mais "des brioches sont des gâteaux", cela voudrait dire que d'autres brioches ne sont pas des gâteaux ». Ce sont bien sûr ces élèves-là qui ont raison... Cela permet à l'enseignant, qui peut s'aider de sa fiche «savoirs du maitre», d'expliquer la différence entre les articles indéfinis spécifiques 
( " un chien est dans la cour », transformation possible en « il y a un chien dans la cour ») et les articles indéfinis génériques («un chien reconnait toujours son maitre», transformation possible en "un chien, ça reconnait toujours son maitre»). Au moyen d'un autre carré-problème proposé la fois suivante, les élèves comprennent alors que le spécifique a bien son pluriel en des ( « des chiens sont dans la cour »), comme on l'apprend à l'école, mais les génériques ont leur pluriel en les, comme les articles définis, eux aussi génériques ( «les chiens reconnaissent toujours leur maitre »). Ce nouveau carré consiste à transformer deux énoncés (« un chien est dans la cour ", « un chien reconnait toujours son maitre ») au moyen d'une double consigne : faire commencer l'énoncé par «il y a un chien qui... » puis recommencer avec " un chien, ça... ». Le débat d'acceptabilité permet de faire le lien avec l'atelier précédent et fixe les deux sens de un en français, chacun ayant son pluriel.

En peu de temps, les élèves ont été invités à réfléchir sur leur langue et à observer son fonctionnement: cette posture métalinguistique, qui passe par une phase de problématisation, se révèle très fructueuse. Nous avons testé ces ateliers dans quatre classes à profil différent au cours de l'année 2013-2014, et les avons fait tester l'année suivante par trois professeurs-stagiaires dans leurs classes. Les ateliers n'ont pas fonctionné dans une de nos classes ni dans une des classes de professeurs-stagiaires, mais ont donné de très bons résultats dans les cinq autres classes, notamment en termes d'intérêt pour la langue chez les élèves en très grande difficulté : ils ont compris qu'ils étaient dans un processus de recherche, d'élaboration en commun d'une règle, et que leurs propos étaient accueillis comme constitutifs de cette règle, ce qui semblait nouveau pour eux.

$\mathrm{Au}$ cours des entretiens que nous avons menés avec les professeurs stagiaires « testeurs ", l'un eux a déclaré :

comment dire, vous voyez quand on fait de la grammaire en classe, ils savent bien qu'on va arriver à quelque chose qui était déjà préconstruit, qui est déjà écrit dans un livre, tout ça. Là, ils m'ont donné l'impression, enfin pas tous, hein, ils m'ont donné l'impression, euh, que voilà, c'est ça, qu'ils pouvaient arriver à quelque chose qui n'était pas écrit d'avance et que ça allait quand même être pris en compte. Voilà c'est ça je crois. Moi j'trouve ça génial, j'ai pas eu cette impression-là autrement, sauf en sciences des fois.

\subsection{Dispositif de recherche et de formation}

38 Les Ateliers que nous proposons ont été testés pendant l'année 2013-2014 au sein d'une équipe de recherche pluricatégorielle composée de conseillers pédagogiques et d'enseignants, ce qui a permis, après avoir soumis les carrés-problèmes à une centaine d'enfants du cycle 3 , de procéder à quelques aménagements. C'est à ce moment que les « ateliers métalinguistiques » au nom barbare pour des enfants sont devenus les « Ateliers d'Antoine », en hommage à A. Culioli.

Dans la logique des propositions de R. Goigoux et S. Cèbe, nous avons proposé pendant l'année 2014-2015 à des étudiants professeurs des écoles stagiaires en deuxième année de master de se saisir de ce dispositif, sans explications supplémentaires ni cours de linguistique sur la TOÉ, afin de nous assurer que des enseignants en situation, ne bénéficiant pas de formation spécifique, pourraient se saisir de cet instrument, le pari étant de modifier les pratiques afin de modifier leurs conceptions, plutôt que l'inverse, habituellement pratiqué mais peu efficace. 

Ils disposaient de 13 Ateliers d'Antoine complets (fiches élèves et "savoirs du maitre ») mis à leur disposition sur un site internet spécifique ${ }^{3}$, uniquement destiné à télécharger les fiches au fur et à mesure que nous les proposions ou modifiions, et à faire remonter leurs impressions. En cours d'année, des entretiens collectifs et individuels ont également permis d'évaluer l'appropriation de l'artéfact. Les entretiens collectifs, sous forme de séminaire, ont permis d'articuler brièvement la dimension cognitive et la dimension sociale des savoirs.

Rappelons que les Ateliers d'Antoine ont été conçus pour être proposés à des petits groupes d'élèves durant les APC, principalement en CM1 et CM2 (enfants âgés de 9 à 11 ans) : c'est donc dans ce cadre que les «maitres-testeurs " sont également intervenus, dans leurs propres classes. stagiaires et demandes d'informations de la part de leurs tuteurs. Les stagiaires impliqués dans la recherche ont été beaucoup plus intéressés par le français que les années précédentes, manifestant eux-mêmes une attitude métalinguistique que nous n'avions pas rencontrée jusqu'à présent dans notre carrière de formateur. Deux des trois maitrestesteurs se déclarent très intéressés pour continuer cette pratique lorsqu'ils seront titulaires et confirment avoir appris de nombreuses notions sur la langue et sur l'enseignement de la langue, alors qu'ils bénéficiaient en parallèle de cours de français «classiques » à l'ESPÉ. La troisième enseignante participant aux tests a abandonné en cours d'expérimentation car elle "ne se retrouvait pas du tout» dans cette façon d'enseigner la langue et était particulièrement gênée par le rapport à la norme scolaire ; elle a exprimé également un conflit de loyauté par rapport à sa formatrice de français.

point le plus important révélé par les entretiens est que ce sont les élèves prétendument les plus en difficulté en "grammaire » qui participent le plus car ils se sentent libérés de la grammaire normative à laquelle ils sont habitués. Ce résultat confirme celui que nous avons constaté en testant nous-même les ateliers (cf. plus haut) : d'une part, les élèves en difficulté, qui sont passifs ou perturbateurs lors des activités traditionnelles, se sont au contraire montrés très actifs et intéressés. D'autre part, ces mêmes élèves ont montré plus d'intérêt pour les leçons dispensées par la suite dans le cadre traditionnel. Il faudrait bien sûr mesurer sur le long terme, dans les productions écrites notamment, ce changement de comportement et de profil des élèves: c'est l'objectif de la nouvelle recherche que nous commençons cette année.

La répartition des formations au sein de l'ESPÉ ne nous a pas permis, cette année-là, d'avoir la responsabilité des cours de français d'un groupe de Master 2 stagiaires. Nous n'avons donc pu travailler qu'avec des enseignants débutants dont nous étions tuteur ou que nous connaissions pour avoir été leur enseignant en Master 1. Cette participation à notre recherche a donc demandé aux volontaires une surcharge de travail et de présence à l'ESPÉ, puisque ce dispositif n'entrait pas dans le cadre officiel de leur formation : deux sur trois étaient cependant très demandeurs et ne regrettent pas du tout cet « investissement » qu'ils jugent « rentable ». 


\section{Conclusion}

45

Notre objectif n'est pas de bouleverser les pratiques des enseignants en classe: pour reprendre les propos de R. Goigoux et S. Cèbe, même si c'est "souhaitable ", ce ne serait pas « raisonnable » car trop éloigné de leurs habitudes de travail ; le but est de proposer aux élèves repérés en difficulté une autre approche de la grammaire, en leur permettant de construire les règles eux-mêmes grâce à la phase de problématisation suivie du débat d'acceptabilité (l'un ne peut pas aller sans l'autre). L'objectif ultime reste bien, à l'école élémentaire, la maitrise du français écrit correct, mais en faisant le pari avec A. Culioli que le travail au niveau métalinguistique stimule l'activité cognitive. Cette approche métalinguistique est d'ailleurs très vivement conseillée, ce qui est nouveau, dans les programmes 2016 du français en cycle 2 et cycle 3. travaillent en petits groupes (le cadre des APC est idéal) et lorsque le travail vient en complément de séances plus traditionnelles effectuées en classe entière.

Au niveau de la formation, il reste à tester le dispositif dans le cadre « habituel » d'un cours de français destiné à des professeurs-stagiaires en Master 2. Nous avons toutefois démontré la pertinence de notre proposition: il est possible que des enseignants débutants, polyvalents et issus d'un cursus non littéraire accèdent à des savoirs linguistiques complexes, se les approprient en «faisant » et non en «écoutant » ou en lisant et, ce qui n'est pas négligeable, s'intéressent ainsi à l'enseignement de la grammaire, faisant ainsi mentir S. Meleuc et N. Fauchard: la grammaire n'est plus le « noyau dur de l'immobilisme » que ces chercheurs dénonçaient en 1999 (p. 15).

\section{BIBLIOGRAPHIE}

BOUQUET, S. (2004). « Antoine Culioli, un linguiste devant les textes saussuriens ». In : Bouquet, S. (éd.), Ferdinand de Saussure. Paris : Éd. de l'Herne, p. 138-149.

BRONCKART, J.-P. (1989). « Du statut des didactiques des matières scolaires ». Langue française 82, p. 53-66.

- (2014), « Des effets en retour de l'analyse didactique ». In : Avanzi, M. et al. (éds), Enseignement $d u$ français : les apports de la recherche en linguistique. Bruxelles : Peter Lang, p. 29-41.

- (2016), «Que faire de la grammaire et comment en faire ?» Pratiques 160-170.

CALAME-GIPPET, F. (2008). «Entrer dans l'analyse grammaticale de la langue à l'école : les ressources ignorées des élèves ». In : Penloup, M.-C. (dir), Les connaissances ignorées; approche pluridisciplinaire de ce que savent les élèves. Paris : INRP, p. 7-104.

CHERVEL, A. (1977). Et il fallut apprendre à écrire à tous les petits Français. Histoire de la grammaire scolaire. Paris : Payot.

CULIOLI, A. (1990-2000). Pour une linguistique de l'énonciation, 3 vol. Gap/Paris : Ophrys.

Pratiques, 169-170 | 2016 
CULIOLI, A. \& NORMAND, C. (2005). Onze rencontres sur le langage et les langues. Gap : Ophrys.

GOIGoux, R. \& CÈBE, S. (2007). « Concevoir un instrument didactique pour améliorer la

compréhension des textes ». Repères 35, p. 185-191.

- (2011), « Un autre rapport entre recherche, pratique et formation. Les instruments didactiques comme vecteur de transformation des pratiques des enseignants confrontés aux difficultés d'apprentissage des élèves ». In : Talbot, L. (éd.), Les pratiques d'enseignement et la prise en compte de l'hétérogénéité des élèves. Bruxelles : De Boeck.

halté J.-F. \& PetitjeAn, A. (dirs) (1980). « Pour un nouvel enseignement du français ». Actes du colloque de Cerisy. Pratiques, numéro spécial.

MELeUC, S. \& FAUCharT, N. (1999). Didactique de la conjugaison. Paris : Bertrand-Lacoste.

RABARDEL, P. (1995). Les hommes et les technologies. Approche cognitive des instruments contemporains. Paris : Armand Colin.

VARGAS, C. (2009). « Peut-on inventer une grammaire pour la réussite scolaire ? » Repères 39, p. 17-39.

\section{NOTES}

1. Français CE1, Hachette, 1995.

2. Nous visons surtout le $\mathrm{CM} 1$ et le $\mathrm{CM} 2$, les deux premières années du cycle 3 français, équivalentes aux $5^{\mathrm{e}}$ et $6^{\mathrm{e}}$ primaires dans lesquelles les élèves ont entre 9 et 11 ans.

3. Ce site n'est pas référencé sur les moteurs de recherche et fonctionne par identifiants : http:// ateliers-antoine.wix.com/ateliers-d-antoine.

\section{RÉSUMÉS}

La plupart des futurs enseignants du premier degré ont une représentation stéréotypée de la grammaire scolaire, qui est un objet construit par l'école ne reposant pas sur un référent théorique solide; pourtant, les sciences du langage ont proposé des façons plus vraies et plus logiques de rendre compte du fonctionnement de la langue. Il est difficile de sensibiliser les futurs enseignants à ces théories linguistiques éloignées de leurs représentations, et de leur en montrer la pertinence. C'est pourquoi nous avons décidé d'associer trois jeunes enseignants à une équipe de recherche qui vise à montrer qu'il est possible de transposer dans l'enseignement du premier degré des théories linguistiques réputées difficiles, comme la théorie des opérations énonciatives d'A. Culioli. En testant dans leurs classes des outils forgés par la recherche (les Ateliers d'Antoine), les jeunes enseignants s'approprient les concepts mieux que si l'on tentait de leur expliquer la théorie.

Most future primary school teachers have a stereotypical representation of the grammar school, which is an object constructed by the school not based on any theoretical referent; however, the language sciences proposed truer and more logical ways to report on the functioning of the language. It is difficult to educate future teachers to these remote linguistic theories of their 
performances, and show them the relevance. That's why I decided to combine three young teachers with a research team that aims to show that it is possible to transpose to the primary education of linguistic theories considered difficult, like the theory of enunciative operations A. Culioli. By testing in their classrooms tools forged through research (the Ateliers d'Antoine), young teachers take ownership of the concepts better than if we tried to explain the theory.

INDEX

Mots-clés : grammaire scolaire, formation des professeurs des écoles, équipe de recherche pluricatégorielle, opérations énonciatives, transposition didactique

Keywords : school grammar, teacher training, mixed research team, enunciative operations, didactic transposition

\section{AUTEUR}

\section{LIONEL AUDION}

